\title{
The geometric theory of elasticity of liquid crystal polymer materials
}

\author{
Theory of liquid crystal elastomers and polymer networks: connection \\ between neoclassical theory and differential geometry \\ Authors: Thanh-Son Nguyen and Jonathan V. Selinger \\ Eur. Phys. J. E (2017) 40: 76.

\begin{abstract}
Recommended with a Commentary by Thomas R. Powers, School of Engineering and Department of Physics, Brown University
\end{abstract}

We say a solid object has strain whenever the distance between two nearby material points is different from the preferred value of this distance. Mathematically, the distances between nearby material points are encoded in the metric tensor. We label material points with coordinates, and describe the configuration of a solid object by an embedding, i.e. the positions in space of the material points. Given the difference in the coordinates for two nearby material points, the metric tensor tells us the distance between the positions of the material points. The metric tensor can change when the object is deformed, so we distinguish between the current metric tensor associated with the actual strain in the object, and the reference metric tensor describing the state of zero strain. Often we think of the reference configuration of an object as the strain-free state achieved when there are no external forces. However, it may be that due to plastic deformation or a change in temperature that there is no way to embed an object so that the reference metric is attained in the absence of external forces. In this case we say the object has residual stress. Residual stress is usually undesirable in engineering applications. However, changing the reference metric can be a convenient way of changing an object's shape without subjecting it to external forces [1]. For example, if one face of a flat thin circular plate is gently heated so that it expands isotropically, the reference metric changes so that the plate deforms into a spherical cap. To get more complicated shapes, it is necessary to prescribe a more complicated pattern of swelling or contraction.

One idea for creating responsive shape-changing materials is to exploit anisotropy. Liquid crystal polymeric materials are ideal for this purpose. These materials consist of rod-like molecules which are cross-linked to a polymer network [2]. In the nematic phase, the rodlike molecules make the material anisotropic, causing swelling (from heating, say) along the rod directors to be different from the swelling in perpendicular to the directors. By heavily cross-linking the material, the director field may be fixed to the polymer network and act as a "blueprint" for a desired swelling pattern [3].

Theory is required to quantitatively predict the swelling pattern for a given blueprint. Much recent work uses a geometric theory of elasticity, in which the change in the reference 
metric tensor upon swelling is determined by lengthening the material along the director and shortening it perpendicular to the director [4]. This approach contrasts with the neoclassical theory of Warner and Terentjev, who generalized the theory of rubber elasticity by treating the polymers in a liquid crystal elastomer as anisotropic random walks [2]. Since the neoclassical theory treats the director and the network as independent degrees freedom, it is appropriate for weakly cross-linked systems. It is natural to ask how these two different problems and the corresponding approaches are related; this question is precisely answered by subject of this commentary, the recent paper by Nguyen and Selinger.

The starting point of Nguyen and Selinger is the neoclassical theory, which they express in the language of differential geometry using the local tangent vectors at the time of crosslinking and at the current time, as well as the director field at the time of cross-linking and at the current time. For a lightly cross-linked liquid crystal elastomer, minimizing the free energy over the current tangent vector and director field yields the shape and director pattern. The shape and the director field are independent degrees of freedom in this case, since for example an external field could cause the director to depart from the value that minimizes the free energy for the given network shape. For a highly cross-linked material, which Nguyen and Selinger call a liquid crystal network, the director is not treated as an independent degree of freedom; instead, it always takes the value that minimizes the free energy for the current shape. Calculating the minimum reveals that the current director field arises from a balance of two competing physical effects. If the nematic order is weak, then the current director field aligns along the principal axis of the network shear. If the nematic order is strong, then the current director aligns with the image in the deformed network of the material line that coincides with the director in the reference state. Furthermore, if there are no external forces acting on the network, then metric tensor that minimizes the energy is precisely the reference metric tensor used in the geometric theory of elasticity. In this case, the competing effects agree, and the director aligns with the principal shear direction, which is the same as the image of the material element along the reference director.

To sum up, the paper by Nguyen and Selinger shows how the theory of liquid crystal polymer networks is related to the theory of the differential swelling of elastic gels. Furthermore, it explicitly shows how to determine the response of a director field to a change in network conformation. These insights can only help to deepen the understanding required to design smart shape-changing materials.

\section{References}

[1] E. Sharon and E. Efrati, Soft Matter 6 (2010) 5693.

[2] M. Warner and E. M. Terentjev, Liquid Crystal Elastomer (Oxford University Press, 2003).

[3] C. D. Modes and M. Warner, Phys. Rev. E 84 (2011) 021711.

[4] C. D. Modes, K. Bhattacharya, M. Warner, Phys. Rev. E 81 (2010) 060701. 